\title{
AN APPROPRIATE FEATURE CLASSIFICATION MODEL USING KOHONEN NETWORK
}

\author{
R. Sridevi \\ Research Scholar, Bharathiar University, Coimbatore, India \\ Dr. P. Dinadayalan \\ Assistant Professor of Computer Science, Department of Computer Science, \\ Kanchi Mamunivar Centre for Post Graduate Studies, Puducherry, India

\section{S. Bastin Britto} \\ Research Scholar, Bharathiar University, Coimbatore, India
}

\begin{abstract}
Self-Organizing Maps are widely used unsupervised neural network architecture to discover group of structures in a dataset. Feature Selection plays a major role in Machine Learning. "An Appropriate Feature Classification Model using Kohonen Network (AFCM)" is based on Recurrent Neural Network approach for feature selection which clusters relevant and irrelevant features from the dataset present in cloud environment. The proposed model not only clusters relevant and irrelevant features but also refine the clustering process by minimizing the errors and irrelevant features. The AFCM consists of Feature Selection Organizer and Convergence SOM. In the Feature Selection Organizer, features are clusters into Relevant and Irrelevant Feature classes. The Convergence SOM helps to improve the prediction accuracy in the Relevant Feature set and to reduce the irrelevant features. The efficiency of the proposed model is extensively tested upon real world medical datasets. The experimental result on standard medical dataset shows that the AFCM is better than the Traditional models.
\end{abstract}

Keywords: Classification, Cloud database, Recurrent Neural Network, SelfOrganizing Map.

Cite this Article: R. Sridevi, Dr. P. Dinadayalan and S. Bastin Britto, An Appropriate Feature Classification Model Using Kohonen Network, International Journal of Computer Engineering and Technology, 10(2), 2019, pp. 148-159.

$\mathrm{http}: / /$ iaeme.com/Home/issue/IJCET?Volume $=10 \&$ Issue $=2$ 


\section{INTRODUCTION}

This chapter focuses on Feature Selection Model, Machine Learning using neural network and Cloud database. Feature selection has been an active research area in pattern recognition, statistics, and data mining communities. The main idea of feature selection is to choose a subset of input variables by eliminating features with little or no predictive information. Feature selection can significantly improve the comprehensibility of the resulting classifier models and often build a model that generalizes better to unseen points [1]. Further, it is often the case that finding the correct subset of predictive features is an important problem in its own right. The major steps involved in a Feature Selection Method are Generation, Evaluation, Stopping Criterion and Validation [2]. Feature selection is the base to machine learning.

Machine learning provides computers with the ability to learn without being explicitly programmed [3]. Machine learning focuses on the development of neural networks that can teach themselves to grow and change when exposed to new data. A neural network is a mathematical model or computational model that tries to simulate the structure and functional aspects of biological neural networks [4]. The neural network demonstrates the behaviour of the Feed Forward network and recurrent network. Real world dataset which contain varying number of features and produce the reduced feature sub set as an output that is also varying in size. So, Feed Forward neural network which have fixed size inputs and outputs, cannot be automatically applied to temporal sequences of arbitrary length.

Recurrent neural network have feedback path from their outputs to the inputs, the response of such network is recursive. Recurrent networks can have signals travelling in both the directions by introducing loops in the network. Recurrent networks are dynamic in nature (i.e) their state is changing continuously until they reach an equilibrium point. They remain at the equilibrium point until the input changes and a new equilibrium needs to be found. Most of the machine learning methods have been used feed forward Kohonon's Self organizing map for feature selection techniques.

The Kohonon's Self Organizing Map has been used for visualization and clustering enormous cloud databases. Feed Forward Neural Network Self-Organizing Map has the characteristics that neuron not only adapt themselves to the data, but also adjust the neighboring neurons as well. It is a type of unsupervised learning which discovers some underlying structure of the data. It also maintains topology-preserving map because there is a topological structure imposed on the nodes in the network.

Cloud database management system is a distributed database that delivers computing as a service. It is sharing of web infrastructure for resources, software and information over a network. The cloud is used as a storage location and database can be accessed and computed from anywhere. (CDBMS)

A cloud database is a database service built and accessed through a cloud platform and access to the database is provided as-a-service. Database services take care of scalability and high availability of the database. Database-as a Service model in Cloud Computing offers many extensive database services. In the proposed model cloud data base offers the services of providing required datasets.

The rest of the paper is organized as follows. Section 2 describes the various feature selection methods and their limitations and also the different areas where the Self Organizing Map is used with respect to the feature selection domain. Section 3 proposes "An Appropriate Feature Classification Model using Kohonen Network (AFCM)" and its components. Section 4 derives Algorithm for AFCM. Section 5 shows the experimental results which are implemented using MATLAB 9.2. Finally Section 6 summarizes the proposed work. 


\section{RELATED WORK}

The selection of relevant features, and the elimination of irrelevant ones, is one of the central problems in machine learning, and many induction algorithms incorporate some approach to addressing it. An irrelevant feature is defined in such a way that it provides no useful information in any context. Irrelevant features can never contribute to prediction accuracy. The relevance measure for a feature goes from strong to weak, the weakest relevancy feature automatically comes under the category of Irrelevant and it never plays a vital role in the classification also.

Feature selection facilitates machine learning (e.g., classification) by aiming to remove irrelevant features. In the presence of many irrelevant features, classification algorithms tend to overfit training data, as too many parameters (corresponding to the number of features) in a learning model would allow the model to adjust to specific random factors in the training data which are not common in the full data. Once overfitting happens, the gap between the estimated and the true accuracy becomes big, and the performance of the classifier can deteriorate significantly. Overfitting poses a serious problem especially for learning on data with high dimensionality.

Some of the existing Feature Selection methods like FCBF, FSBAR [5], and FRFS[6] use different measures to remove irrelevant features. Feature weighting techniques may have difficulties when irrelevant features are present. By removing irrelevant information, dimensionality of the problem is reduced and classification performance is improved.

Feature Selection Methods are used to reduce the number of feature set in training set which in turn effectively reduce the use of computing resources. Removing noisy features from the dataset using Feature Selection Methods improve the classification accuracy of the Classification Algorithm also.

L.Yu et al.[7] surveys existing feature selection algorithms for classification and clustering. They group and compare different algorithms with a categorizing framework based on search strategies.

Feature ranking algorithm was proposed by K.Javad et al.[8] which computes the weights, used for feature ranking, more efficiently as compared to the mutual information measure [9]. It uses a filtrapper approach to select a final subset. When this approach is introduced as a preprocessing step to the Feature subset selection algorithms, Class Dependent Feature Extraction not only presents them with a feature subset, but also relieves them from heavy computations.

M.Dash et al.[10] proposed a method called RELIEF-C for unlabeled data to select relevant features for clustering. The drawback present in the Forward and Backward selection that is if even one feature is selected (or rejected as in the case with backward selection) wrongly in the early stage of selection of features the rest of the choices are negatively affected in a cascaded manner. This method modifies RELIEF so as to overcome its inherent difficulties in the presence of large number of irrelevant features and/or significant number of noisy tuples.

The methods based on heuristics [11] that do not help when there are redundant attributes and most of the methods for dealing with noisy example have embedded the selection process within the learning algorithm. By identifying examples that are non typical in the determination of relevant, irredundant attributes, irrelevant examples can be eliminated prior to the learning process.

A three-step algorithm was proposed by J.Bins et al.[12] which performs feature selection from huge feature sets by removing lots of irrelevant and redundant features. In this algorithm, the first step uses Relief algorithm to remove irrelevance; the second step clusters 
features using K-means to remove redundancy; and the third step is a standard combinatorial feature selection algorithm.

The feature redundancy has been handled in [13] which provides a framework that begins with a conventional combinatorial optimization formulation for similarity preserving feature selection that assess features capability in preserving sample similarity, which can be inferred from either label information or predefined distance metric.

A squared correlation function is employed as the criterion to measure the dependency between features and the features are selected in a stepwise way, by estimating the capability of each specified candidate feature subset to represent the overall features in the measurement space The disadvantage of this type of approach is that it assumes that a linear relationship exists between sample features but in many cases, where features are linked by some nonlinear relationship, this assumption may become unreasonable[14].

According to the idea of mRMR, incremental selection of feature does not provide a mechanism to remove potentially redundant features from the already selected features. This drawback is overcome by a two-stage feature selection algorithm [15] which derives mRMR(Maximum Relevance and Minimum Redundancy) for first -order incremental feature selection and combine $\mathrm{mRMR}$ with feature selectors.

Using the maximum margin principle the discriminative features were found in semisupervised embedded feature selection method [16]. This method identifies an optimal subset of features by maximizing a performance measure that combines classification margin with manifold regularization.

HyunsooYoon et al.[17] presents a new neural network approach, which is called an algorithm learning based neural network (ALBNN), to produce new relevant features and to improve classification accuracy by integrating feature selection and classification procedures. The coevolution learning (with discrete and continuous representations) is used to select relevant features in evolutionary computing [18] by minimizing the neural configuration. The MLP-based feature selection method [19] uses neural network as Classifier and selects the most important features.

Since SOFM (Self Organizing Feature Map) has many useful properties such as topological ordering, density matching and feature selection, it has been widely applied in the areas like Image Classification, Medical, Stock Market and Speech recognition.

SOFM Neural Network applied in clustering analysis [20] had better adaptability for image registration tasks. The Dynamic SOFM classification process is used as a character classification before the conversion of the handwritten image into machine readable format [21]. A change detection of aerial images algorithm based on Kohonen Self-Organizing maps [22] is applied to detect changes in Amazon forest. Self-Organizing Map has been used frequently to cluster and classify satellite images that concerns reflectance spectra of ocean waters [23]. The co-occurrence matrix is used in order to well describe the texture feature of images in an Information retrieval algorithm based on knowledge discovery and SelfOrganizing feature map neural network (SOFMNN) which aim at the defection with slow speed of image information retrieval.

A SOM-based method [24] is used for classifying and predicting the data of female subjects taking the specific health examination and this method considers the subjects with unhealthy-level visceral fat to be targets. Jana Tuckova [25] applied alternatives in SelfOrganizing Map namely KSOM \& SSOM in the improvement of diagnostics and to ensuring the improvement in the treatment of both children and adult patients suffering from diseases affecting social interaction. The Neural Network algorithms like the Kohonen Self-Organizing Map, learning vector quantification and the multilayer perceptron are used in classification 
[26] for the analysis of EMG data recorded during gait. Kohonen Self-Organizing Map is used to increase the efficiency of mapping process in the classification of the speech signals using the objective speech quality measure.

Self-Organizing Map is also applied to analyze the stock market data [27] and to investigate historical information on past human adverse events in an active war region. A Two-Stage Self-Organizing Map is used for detecting and removing the abnormal data-noise from each cluster [28]. An approach latent semantic indexing of 1D-Self-Organizing Map can be used to reduce the dimensionality of document vectors without essentially losing information contained in the full vocabulary. Richard T.Freeman et al.[29] presents a method for content management and knowledge discovery using a topology-preserving neural network and this method provides a natural way to automatically generate topic directories and structures for efficient information access and retrieval.

Chi-Hsu Wang et al.[30] designed a new RNN controller with SOM to force an agent (or defending missile) toward a target (or incoming missile), and a monitoring controller is also designed to reduce the error between RNN controller and ideal controller. One of the SelfOrganizing Map neural network's applications is clustering of animals based on their features [31]. A classifier Self-Organizing Map-INN that combines Self-Organizing Map Neural Network with INN( Informative Nearest Neighbors)[32] is used to reduce the classification time of the unknown object, considering that the time factor is of utmost importance for real time applications. R.Sridevi et al.[33] proposed a Feature Selection Model which removes irrelevant, redundant, noisy data and retains relevant, correlated features in Cloud environment with Feature Interaction. This model consists of Dynamic Self-Organizing Feature map and Feature Associative Memory.

From the literature survey, it is understood that most of the existing classification algorithms [7,17] tend to perform badly in a situation of many irrelevant features. Since the proposed model has to handle irrelevant features, conventional classification algorithms cannot be used as a classifier which is very sensitive to noisy training data and can degrade their performance due to overfitting and poor generalization. The existing classification algorithms [21,24] also have the drawback as they do not optimize the configuration of the classifier in selecting features.

Most of the conventional Feature Selection methods fail to handle the relevant and irrelevant features efficiently and there is no chance of recursive paths in these methods. The process of clustering the relevant and irrelevant features in the feature set can be finely tuned through the recursive paths provided by recurrent neural network.

Thus, we propose a new model "An Appropriate Feature Classification Model using Kohonen Network (AFCM)" which clusters relevant and irrelevant features from the dataset taken from the Cloud databases. An Algorithm is also derived for this model which accepts both relevant and irrelevant features present in the dataset as an input and produces the relevant feature sets alone as the output.

\section{ARCHITECTURE}

From the literature survey, the Feature Selection methods like [6,11] select the most relevant features using different approaches but does not project high-dimensional data onto lowdimensional grids. In some learning algorithms [7, 8] irrelevant features are removed in stepwise manner but fail to preserve the topological relationship of the training samples that is data which are close in the input space are mapped into neural nodes which are also close in the grids.

Feature Selection method with Multilayer Perceptrons [13] does not support non-linear model which makes it available to process complex real world data and this method fails to 
handle high dimensional data efficiently. The conventional feature selection method $[6,8]$ does not provide solution for the dataset which contain some missing data and for non-linear mapping of datasets.

Most of the existing Self-Organizing Map-based methods [14,16] use Feed Forward Neural Network Self-Organizing Map for classification and clustering process, fail to handle relevant and irrelevant features, reduce prediction accuracy and lead to overfitting which causes a degradation in learning especially on high dimensional data. To overcome the limitations present in the conventional feature selection methods, we propose "An Appropriate Feature Classification Model using Kohonen Network (AFCM)" which is used to group similar features and replace them by a single representative. Since the proposed model is Recurrent Neural Network SOM-based, it is topologically constrained, less prone to local minima than the traditional Feature Selection Methods.

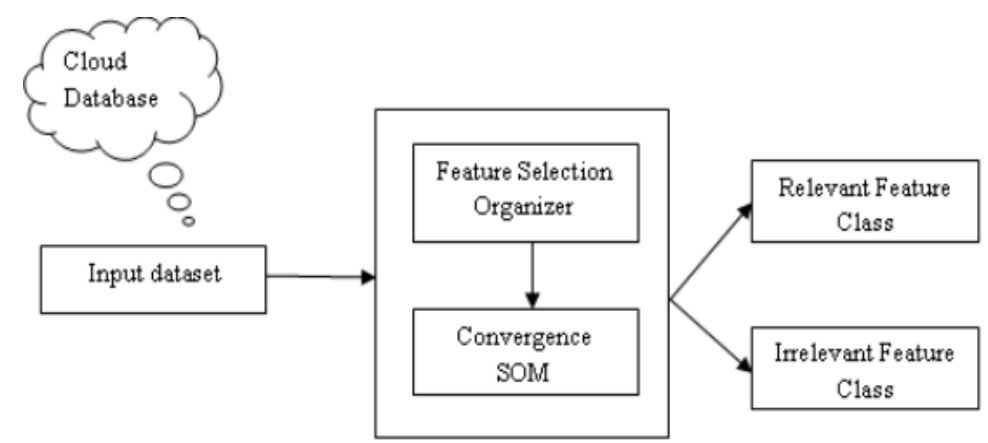

Figure 1 Architecture of AFCM

The AFCM is a machine learning technique which is implemented by using Recurrent Neural Network. The structure of the proposed model is a Recurrent Neural Network based Self-Organizing Map which is dynamic in nature (i.e) their state is continuously changing until an equilibrium point is reached. They remain at the equilibrium point until the input changes and a new equilibrium needs to be found. The proposed model also supports unsupervised learning so that datasets with unlabeled classes can also be efficiently clustered into relevant and irrelevant feature class. Figure 1 demonstrates the architecture of AFCM which groups relevant and irrelevant features from cloud database. It comprises Feature Selection Organizer and Convergence SOM. Feature Selection Organizer classifies Relevant and Irrelevant features through mapping process. Mapping process is based on Forward Finite Difference method. The structure of Feature Selection Organizer is in the form of Kohonen Self-Organizing Map and uses unsupervised learning. Let the training dataset (TD) from the Cloud Database consists of Schema $\mathrm{T}=\{\mathrm{A} 1, \mathrm{~A} 2, \ldots \mathrm{An}\}$ where $\{\mathrm{A} 1, \mathrm{~A} 2, \ldots \mathrm{An}\}$ are features and let $\mathrm{X}=\{\mathrm{X} 1, \mathrm{X} 2, \ldots \mathrm{Xn}\}$ be the instance of schema $\mathrm{T}$ which are used as input vectors for Feature Selection Organizer. Using Forward Finite Difference method, Feature Selection Organizer trains the given cloud dataset. During the training, the Relevant and Irrelevant features are partitioned into Relevant Feature Class (RFC) and Irrelevant Feature Class (IFC) such as $\mathrm{RFC}=\left\{\mathrm{RF}_{1}, \mathrm{RF}_{2}, \ldots, \mathrm{RF}_{\mathrm{p}}\right\}$ and $\mathrm{IFC}=\left\{\mathrm{IF}_{1}, \mathrm{IF}_{2}, \ldots, \mathrm{IF}_{\mathrm{q}}\right\}$. Relevant Feature Class (RFC) is given as an input to Convergence SOM. Eventually Convergence SOM measures quality of relevant feature of the cloud database and also preserves neighbourhood relationship of cloud dataset. The structure of Convergence SOM is a similar structure of Feature Selection Organizer which uses Central Finite Difference Method. Convergence SOM is used to minimize the average distance between each feature vector and its BMU (Best Matching Unit) so that Relevant Features in RFC will be mapped to nearby locations on the map. 


\subsection{Feature Selection Organizer}

Feature Selection Organizer identifies relevant features using Forward Finite Difference Method. During training, a mapping from class $C$ to input vector $X$ which is the instance of schema $\mathrm{T}$ is created based on Forward Finite Difference Method and features are partitioned into two classes namely Relevant Feature Class (RFC) and Irrelevant Feature Class (IFC) such as $\mathrm{RFC}=\left\{\mathrm{RF}_{1}, \mathrm{RF}_{2}, \ldots, \mathrm{RF}_{\mathrm{p}}\right\}$ and $\mathrm{IFC}=\left\{\mathrm{IF}_{1}, \mathrm{IF}_{2}, \ldots, \mathrm{IF}_{\mathrm{q}}\right\}$.

The Feature Selection Organizer proceeds on two steps namely Initialization and Training. In the Initialization step, the input feature vectors of instances $\{\mathrm{X} 1, \mathrm{X} 2, \ldots . \mathrm{Xn}\}$ are taken as an input from the cloud dataset. At time $\mathrm{t}=0$, randomly initialize a value to $\mathrm{w}_{\mathrm{ij}}$ for the neuron in position $(\mathrm{i}, \mathrm{j})$ for each input feature vector $\left\{\mathrm{F}_{1}, \mathrm{~F}_{2}, \ldots \mathrm{F}_{\mathrm{n}}\right\}$. The Training step calculates the relevancy difference between two consecutive features using Forward Finite Difference Method. Let the relevancy difference between two consecutive features namely $F_{i}$ and $F_{i+1}$ be denoted by $Y_{i}$ and defined by $Y_{i}=\Delta\left(F_{i+1}-F_{i}\right)$ for $i=1$ to $n$.

This relevancy difference is summed up at first stage by using (1).

$\mathrm{S}_{1}=\sum_{i=1}^{n} \mathrm{Yi}$ where $\mathrm{Yi}=\Delta\left(\mathrm{F}_{\mathrm{i}+1}-\mathrm{F}_{\mathrm{i}}\right)$

In the consecutive stages that is from $\mathrm{S}_{\mathrm{i}}$ for $\mathrm{i}=2$ to $\mathrm{n}$, relevancy difference between two consecutive $\mathrm{Y}_{\mathrm{j}}$ 's is calculated using (2).

$\mathrm{S}_{\mathrm{i}}=\sum_{j=1}^{n} \mathrm{Yj}$ where $\mathrm{Y}_{\mathrm{j}}=\Delta\left(\mathrm{Y}_{\mathrm{j}+1}-\mathrm{Y}_{\mathrm{j}}\right)$

At time $\mathrm{t}>0$, the weights of output neuron $\mathrm{j}$ and its adjacent neurons should be corrected using (3).

$$
\mathrm{w}_{\mathrm{ij}}(\mathrm{t}+1)=\mathrm{w}_{\mathrm{ij}}(\mathrm{t})+\alpha(\mathrm{t})\left[\mathrm{F}_{\mathrm{i}}(\mathrm{t})-\mathrm{w}_{\mathrm{ij}}(\mathrm{t})\right]
$$

where $\alpha$ is a learning rate. The value of $\alpha$ will decrease to 0 with the increase of time. In generally, $\alpha$ is set as $\alpha(\mathrm{t})=1 / \mathrm{t}$.

During training, the input feature vectors $\left\{\mathrm{F} 1, \mathrm{~F} 2, \ldots \mathrm{F}_{\mathrm{n}}\right\}$ are partitioned into Relevant Feature Class (RFC) and Irrelevant Feature Class (IFC) such as $R F C=\left\{R F_{1}, R F_{2}, \ldots, R F_{p}\right\}$ and $\mathrm{IFC}=\left\{\mathrm{IF}_{1}, \mathrm{IF}_{2}, \ldots, \mathrm{IF}_{\mathrm{q}}\right\}$.

In order to map the similar features to the nearby locations, the Relevant Feature Class $\mathrm{RFC}=\left\{\mathrm{RF}_{1}, \mathrm{RF}_{2}, \ldots, \mathrm{RF}_{\mathrm{p}}\right\}$ is sent to Convergence SOM.

\subsection{Convergence SOM}

Convergence SOM aims to improve the prediction accuracy and produce error free features. The quality of Relevant Feature Class (RFC) is improved by minimizing average distance between each feature vector and its BMU (Best Matching Unit) in that Class.

During training, the Relevant Feature Class $\mathrm{RFC}=\left\{\mathrm{RF}_{1}, \mathrm{RF}_{2}, \ldots, \mathrm{RF}_{\mathrm{p}}\right\}$ is taken as an input from Feature Selection Organizer. The Central relevancy difference between the features in Relevant Feature Class is denoted by $\delta$ and it is calculated using (4).

$$
\delta \mathrm{RF}_{\mathrm{x}}=\mathrm{RF}_{\mathrm{x}+\mathrm{h} / 2}-\mathrm{RF}_{\mathrm{x}-\mathrm{h} / 2}
$$

where

$\mathrm{h}$ - Interval between the features.

The distance between $\delta \mathrm{RF}_{\mathrm{x}}$ of each feature vector in Relevant Feature Class and its BMU feature is calculated using (5) by applying Central Finite Difference Method.

$$
\mathrm{d}(\mathrm{t}+1)=\mathrm{d}(\mathrm{t}) \delta \mathrm{RF}_{\mathrm{x}}(\mathrm{t})
$$

By minimizing the average distance between the $\delta R F_{x}$ of each feature vector in Relevant Feature Class and its BMU feature, the quality of Relevant Feature Class (RFC) is improved. 


\section{ALGORITHM}

In continuation with the Architecture demonstrates in the Figure 1, the following algorithm is derived to cluster relevant and irrelevant features from the datasets which is retrieved from cloud database offered by Amazon's AWS.

\section{Input:}

D - Dataset from Cloud Database

$\mathrm{n}$ - Number of Features

Output: Relevancy Feature Class RFC

\section{Method:}

Step-1: Let Schema $T=\left\{A_{1}, A_{2} \ldots A_{n}\right\}$ consists of set of features $\left\{A_{1}, A_{2} \ldots A_{n}\right\}$ from $D$.

Step-2: Initialize Relevant Feature Class RFC to empty.

Step-3: At time $t=0$, Set the Learning rate $\alpha(n)$ and neighborhood radius $r(n)$ to predefined constant.

Step-4: At time $\mathrm{t}>0$, Repeat Step-5 until the neural network model is trained by giving input vectors $X=\left\{X_{1}, X_{2} \ldots X_{n}\right\}$ which is the instance of $T$.

Step-5: For each Input Vector $X$ in $T$

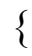

Step-5.1: for each $F_{i}$ in Input Vector $X$

\section{\{}

Step-5.1.1: Repeat Step-5.1.2 to Step-5.1.8 until neural network model partitioned features into Relevant Feature Class (RFC) and Irrelevant Feature Class (IFC) using Feature Selection Organizer.

Step-5.1.2: Calculate the relevancy of the feature $\mathrm{F}_{\mathrm{i}}$ to the target class label $\mathrm{C}$ using the Forward Finite Difference formula $f^{\prime}\left(F_{i}, C\right)=\left(f\left(F_{i+1}, C\right)-f\left(F_{i}, C\right)\right) / Y i$ where $Y i=\Delta\left(F_{i+1}-F_{i}\right)$

Step-5.1.3: Let the relevancy difference between two consecutive features namely $F_{i}$ and $F_{i+1}$ be denoted by $\mathrm{RD}_{\mathrm{i}}$.

Step-5.1.4: Calculate $\mathrm{RD}_{\mathrm{i}}=\Delta\left(\mathrm{f}^{\prime}\left(\mathrm{F}_{\mathrm{i}}, \mathrm{C}\right)-\mathrm{f}^{\prime}\left(\mathrm{F}_{\mathrm{i}+1}, \mathrm{C}\right)\right)$

Step-5.1.5: Calculate the Relevancy Difference at stage $\mathrm{s}$ as $\mathrm{RD}_{\mathrm{s}}=\sum_{i=1}^{s} R D i$

Step-5.1.6: Update Relevancy Difference using the below formula

$$
\Delta \mathrm{RD}_{\mathrm{i}}=\mathrm{RD}_{\mathrm{i}}^{(\mathrm{s})}-\mathrm{RD}_{\mathrm{i}}^{(\mathrm{s}-1)}
$$

Step-5.1.7: Update Relevancy Feature Class RFC (i.e) RFC $=$ RFC U F $F_{i}$

Step-5.1.8: Decrease Learning rate $\alpha(n)$

and increase neighborhood radius $\mathrm{r}(\mathrm{n})$ by 1

\}

Step-5.2: Repeat Step 5.1 until $\delta(\mathrm{RD})>0$ during Convergence SOM. \}

Step-6: Output the Relevant Feature Class RFC.

The proposed algorithm aims to remove overfitting and to improve high classification accuracy effectively. Since the proposed algorithm is based on Self-Organizing Map using $\mathrm{RNN}$, it preserves the topological ordering (i.e) does not affect the neighborhood relationship during training and reduces the complex dataset into simplest dataset. Optimization is 
achieved in terms of space and time complexity by using the proposed algorithm which provides error free (i.e) noiseless features.

\section{EXPERIMENT \& RESULTS}

For experimental purpose, we have taken Heart Disease medical dataset from AWS (Amazon Web Service) Cloud database. For simulation, we have tested four AWS cloud medical databases which were Cleveland.data, Hungarian.data, long-beach-va.data and Switzerland.data. These medical datasets were collected from different cloud locations such as Cleveland Clinic Foundation, Hungarian Institute of Cardiology, Budapest, V.A. Medical Center, Long Beach, CA, University Hospital, Zurich, Switzerland. The proposed AFCM classifies relevant and irrelevant features in Heart Disease medical dataset from AWS Cloud database. The simulation was conducted using Neural Net Clustering App available under neural network toolbox 10.0 in Matlab. The Cleveland database contains 700 instances, Hungarian database contains 600 instances, Switzerland database contains 400 instances, and Long Beach VA contains 500 instances. These medical cloud databases have 76 attributes. The attributes are age, sex, chest_pain_type, resting_blood_pressure, serum_cholestoral, family_history, max_heart_rate_achieved, resting_heart_rate and so on. These attributes are classified as data types like continuous, nominal and integer.

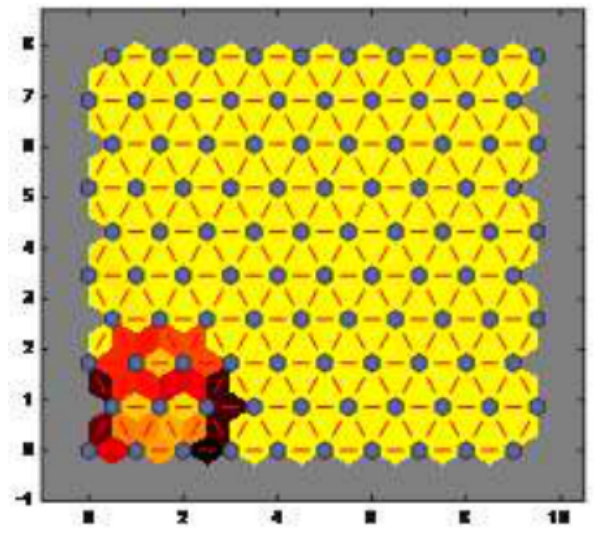

Figure 2 Experimental result of AFCM

The simulation of AFCM is demonstrated in Figure 2. The AFCM measures the similar and dissimilar feature classes in the given cloud dataset using Forward Finite Difference method. It is implemented using Self-Organizing Map. The Relevant and Irrelevant features are divided into RFC and IFC classes. The Forward Finite Difference method calculates the distance between each attribute in Medical Cloud dataset and its Best Matching Classes. The blue hexagons represent the attributes of Medical Heart Disease dataset and red lines connect neighboring attributes. The colors in the regions containing red lines indicate the distances between the Cloud Heart Disease attributes. The darker color indicates larger distance and minimizes a gap between the attribute values in the Cloud datasets. The lighter color uses smaller distances which imply that the attributes are close to each other in the Cloud dataset. The distance between the adjacent attributes in Heart Disease Cloud dataset is calculated using Forward Finite Difference method. The light color areas are viewed as relevant features and dark color areas as Irrelevant features.

During the simulation, the relevant features like Age, Sex, Chol, Trestbps and Restecg in Heart disease database come under Relevant Feature Class (RFC). At the same time, the irrelevant features like Restckm and Exerckm come under Irrelevant Feature Class (IFC) which is shown in the lower left corner of Figure 2 as dark colors. The remaining region (excluding irrelevant features) represents relevant features. Thus "An Appropriate Feature 
Classification Model using Kohonen Network (AFCM)" has successfully classified relevant features which are related to Heart disease.

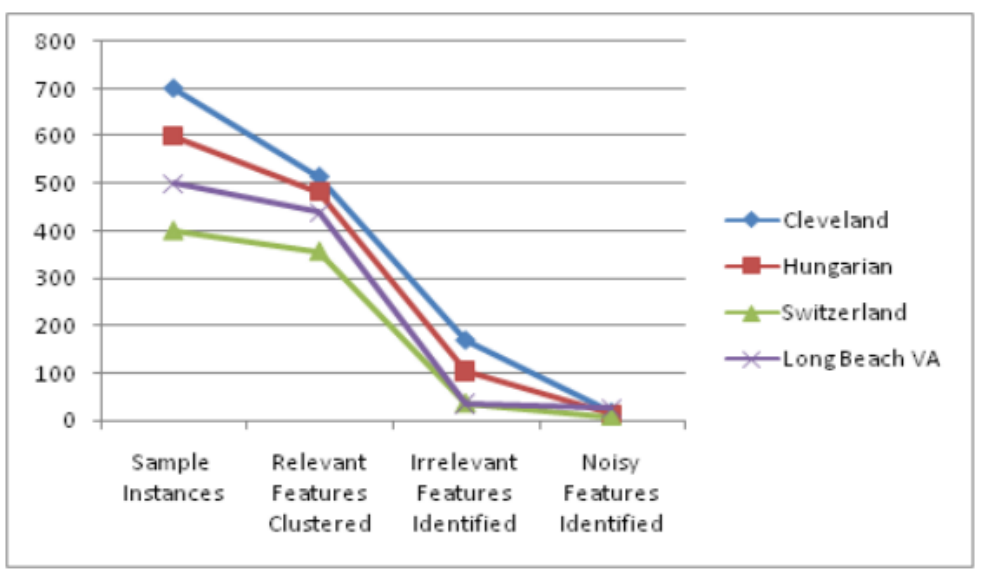

Figure 3 Performance of AFCM

The performance result of AFCM is represented in Figure-3. We have taken 2200 sample inputs from medical cloud Heart Disease dataset for experimental purpose. Out of 2200 instances, 700 instances, 600 instances, 400 instances and 500 instances are captured from Cleveland, Hungarian, Switzerland, Long beach va medical cloud databases respectively. From the Figure-3, the proposed model clusters Relevant, Irrelevant and Noisy features. During the implementation, the relevant features like Age, Sex, Chol, Trestbps and Restecg in Heart disease database come under Relevant Feature Class (RFC). At the same time, the irrelevant features like Restckm and Exerckm come under Irrelevant Feature Class (IFC). These sample instances have been trained and tested successfully. The performance result shows that the percentage of classification of success rate is greater than $97 \%$ for Relevant and Irrelevant features of Heart Disease Medical dataset. Thus "An Appropriate Feature Classification Model using Kohonen Network (AFCM)" has successfully classified relevant features which are related to Heart disease.

\section{CONCLUSIONS}

"An Appropriate Feature Classification Model using Kohonen Network (AFCM)" is a new Feature Classification Model using Kohonen Network which clusters relevant and irrelevant features. The proposed Model is compared with the other existing feature selection methods to overcome the limitation of the later. This model comprises of Feature Selection Organizer and Convergence SOM. The Feature Selection Organizer is clustered into two different classes which has Relevant Feature Class and Irrelevant Feature Class. The Convergence SOM is used to improve the quality of Relevant Features in Relevant Feature Class. The structure of the proposed model is implemented using Self-Organizing Map. The result shows that the proposed AFCM is significantly better than the conventional Feature Selection Methods.

\section{REFERENCES}

[1] Verma,L.K.,Verma,K. and Tripathi,P., Feature Selection Technique using Homogeneity based cluster, International Journal of Engineering Research and General Science, $3(1), 2015$.

[2] Dash,M. and Liu,H., Feature Selection for classification, Intelligent Data Analysis: An Int'lJ., 1(3), 1997, pp.131-156,.

[3] https:// www.expertsystem.com/machine-learning-definition 
[4] https:// www.sciencedirect.com/topics /neuro science/artificial-neural-network

[5] Xie,J., Wu,J. and Qian,Q., Feature selection algorithm based on association rules mining method, In Proceedings of 2009 Eighth IEEE/ACIS International Conference on Computer and Information Science, IEEE, 2009, pp.357-362.

[6] Wang,G., Song,Q., Baowen and Yuming Zhou, Selecting feature subset for high dimensional data via the propositional FOIL rules, Pattern Recognition,46, 2013, pp.199214.

[7] Yu,L. and Liu,H., Toward integrating feature selection algorithm for classification and clustering, IEEE Trans. Knowl. Data Eng., 17 (4), 2005, pp.491-502.

[8] Kashif Javed, Haroon A. Babri and Mehreen saeed, Feature Selection based on ClassDependent Densities for High Dimensional Binary Data, IEEE Transactions on Knowledge and Data Engineering,24(3),2012, (www.Computer.org/csdl/trans/tk/2012/03/ttk2012030465-abs.html)

[9] Jihong Liu, A Hybrid Feature Selection Algorithm for Data set of thousands of variables, IEEE, 2010.

[10] Dash,M. and Ong, Y.S., RELIEF-C: Efficient Feature Selection for clustering over Noisy Data, in : Proc. 23rd IEEE International Conference on Tools with Artificial Intelligence (ICTAI), Roca Raton, Florida, USA, 2011,pp.869-872.

[11] Lashkia,G., Anthony,L., Relevant, irredundant feature selection and noisy example elimination, IEEE Trans syst Man cybern B cybern ,34(2), 2004, pp.888-897.

[12] Bins,J. and Draper,B.A, Feature Selection from huge feature sets, in: Proc. 8th International Conference on Computer Vision (ICCV-01), VanConver, British Columbia, Canada, IEEE Computer Society, 2001,pp.159-65.

[13] Zhao,Z., Liu,H., On Similarity Preserving Feature Selection, IEEE Transaction on Knowledge and Data Engineering, 25(3),2013.

[14] Wei,H.L. and Billings,S.A., Feature subset selection and ranking for data dimensionality reduction, IEEE Trans Pattern Analysis and Machine Intelligence, 29(1), 2007,pp.162166.

[15] Peng,H., Fulmi long, and Ding,C., Feature selection based on mutual information criteria of max-dependency, max-relevance, and min-redundancy, Pattern Analysis and Machine Intelligence, IEEE, 2005, pp. $1226-1238$.

[16] Xu,Z., King,I. and Lyu,M.R.T, Discriminative Semi-supervised Feature Selection via Manifold Regularization, IEEE Trans. Neural Networks, 21(7), 2010,pp.1033-1047.

[17] Hyunsoo Yoon, Cheong-Sool Park, Jun Seok Kim and Jun-Geol Baek, Algorithm learning based neural network integrating feature selection and classification, Elsevier, Expert Systems with Applications,2013,pp. 231-241.

[18] Yamina Mohamed Ben Ali, Advances in evolutionary feature selection neural networks with co-evolution learning, Neural computing and Application , 17, 2008, pp. 217-226.

[19] Enrique Romero and Josep Maria Sopena, Performing Feature Selection with Multilayer Perceptrons, IEEE Transactions on Neural Networks, 19 (3), 2008, pp.431-441.

[20] Ge, L.L., Wu, Y.H., Hua, B., Chen, Z., and Chen, L., Image Registration Based on SOFM Neural Network Clustering, in : Proc. 36th Chinese Control Conference, Dalian, China, 2017, pp. 6016-6020.

[21] Benny, D. , Soumya, K.R. and Nageswara Rao, K., New Dynamic Self-organizing Feature maps for the classification of Extracted Feature vectors of characters, in : Proc. International conference on Robotics, Automation, Control and Embedded system RACE 2015, Hindustan University, Chennai, India, ISBN: 978-81-925974-3-0, 2015. 
[22] Mota,R.L.M. , Ramos,C.B. and Elcio H. Shiguemori, Application of Self-organizing Maps at Change Detection in Amazon Forest, in : Proc. 11th International Conference on Information Technology: New Generation, 2014, pp. 371-376.

[23] Lobo,V.J.A.S, Application of Self-Organizing Maps to the Maritime Environment, Springer-Verlog Berlin Heidelberg, 2009, pp.19-36.

[24] Kamiura,N.S., Kobashi, Nii,M. and Sorachi,K., Application of Self-Organizing maps to data classification and data prediction for female subjects with unhealthy -Level Visceral Fat, in: Proc. IEEE International Conference on systems, man and cybernetics, Budapest, Hungary, 2016, pp.1815-1820.

[25] Tuckova, J., The possibility of Kohonen Self-Organizing Map Applications in Medicine, IEEE, 2013.

[26] Nair,S., French,R.M., Laroche, D. and Thomas, E., The Application of Machine Learning Algorithms to the analysis of Electromyographic Patterns from Arthritic Patients, IEEE Trans. On Neural systems and Rehabilitation Engineering, 18(2), 2010.

[27] Kossakowski,P. and Bilski,P., Application of Self-Organizing Maps to the Stock Exchange Data Analysis, in: Proc. 8th IEEE International conference on Intelligent Data Acquisition and Advanced Computing Systems Technology and Applications, warsaw Poland, 2015,pp.208-213.

[28] Apinantanakon,W. and Sunat,K., A two-stage SOM for noise reduction in SOM, in : Proc, IEEE International Computer Science and Engineering(ICSEC), 2014, pp.198-202.

[29] Freeman,R.T. and Yin,H., Web Content Management by Self-Organization, IEEE Trans. On Neural Networks, 16(5),2005.

[30] Wang,C.H., Cychen and Hung,K.N., Toward a New Task Assignment and Path Evolution (TAPE) for Missile Defence System(MDS) using Intelligent Adaptive SOM with Recurrent Neural Networks(RNNs), IEEE Trans on Cybernetics,45(6),2015.

[31] Behbahani,S. and Nasrabadi,A.M., Application of SOM neural network in clustering, $J$. Biomedical Science and Engineering, 2009, pp.637-643.

[32] Moreira,L.J. and Silva,L.A., Data Classification combining Self-organizing Maps and Informative Nearest Neighbor, International Joint Conference on Neural Network(IJCNN), 2016, pp.706-713.

[33] Sridevi,R. and Dinadayalan,P., A Dynamic Neural Feature Selection Model using Cloud Databases, Australian Journal of Basic and Applied Science.10(12),2016,pp.117-125. 